\section{Après la catastrophe: le temps de la prise en charge psychologique des victimes}

\author{
P.-A. Savary
}

"Si certains, par quelque capacité d'oubli ou de résistance, semblent traverser à l'envers l'Achéron sans trop de difficultés, d'autres en gardent une blessure profonde. C'est eux que nous tentons de comprendre, voire de soigner."

C. Barrois

L'auteur passe en revue les phénomènes psychologiques survenant au décours d'une catastrophe ainsi que les mesures à prendre au cours des différents stades à travers lesquels les victimes, leurs proches et les intervenants peuvent passer. Il précise aussi les conditions qui imposent le recours à un spécialiste afin de prévenir l'apparition d'un état de stress post-traumatique et d'autres comorbidités.

\section{Les effets de la catastrophe sur le psychisme}

Comme les terribles et saisissantes images télévisées nous l'ont abondamment démontré, une catastrophe crée le chaos, et sans ce chaos, on ne saurait parler de catastrophe.

Au cours de ces événements, des êtres humains sont brutalement confrontés, éprouvent dans leur chair et leur âme des situations effroyables, qui dépassent en intensité tout ce qui avait pu leur arriver jusqu'alors.

Les interventions viseront donc à organiser progressivement ce chaos pour leur porter secours avec un maximum d'efficacité. Pour les victimes, la catas-

Dr Pierre-Alain Savary

Spécialiste FMH en psychiatrie et psychothérapie

Membre fondateur de la Société Suisse de Psychotraumatologie

Chemin de Pré-Fleuri 17

CH-1260 Nyon

E-mail: pasavary@vtx.ch
La Société Suisse de Psychotraumatologie, société affiliée à la Société Suisse de Psychiatrie, a pour but principal l'étude des traumatismes psychique et de leur traitement, l'information de ses membres sur tous les problèmes liés au stress traumatique que cela soit par rapport au personnel soignant ou aux patients, de diffuser des informations préventives auprès de la population en général et des personnels intervenants. La société a également pour but de créer des espaces de réflexion et d'échange sur le thème des traumatismes psychiques. Elle offre un lieu de formation continue par l'organisation de conférences et de séminaires. Elle organise un groupe d'action pour informer les diverses institutions compétentes et les autorités sur les traumatismes psychiques.

Elle dispose depuis peu d'un petit site Internet (www.sspt.net).

trophe entraîne une atteinte bio-psycho-sociale. Comme on a pu le constater, non seulement la complexité interne des individus est touchée, voire détruite, mais les multiples systèmes et réseaux qui caractérisent nos sociétés modernes, la collectivité dans laquelle nous vivons, sont eux aussi atteints.

Les catastrophes atteignent 3 catégories de population: les victimes directement impliquées, les intervenants (pompiers, sauveteurs ainsi que toute la chaîne sanitaire jusque et y compris les médecins hospitaliers) et les proches des victimes (famille directe, partenaire, amis, collègues).

Les membres des deux premiers groupes sont susceptibles d'être victime d'un traumatisme psychique, et ceci d'autant plus que leur atteinte somatique aura été légère.

Les facteurs déclenchants de la traumatisation sont avant tout liés au fait que la victime éprouve de la terreur, lors d'un incident unique et inattendu, dont la soudaineté et la violence submerge en lui toute possibilité de maîtrise immédiate. Cette intrusion brutale et violente d'une réalité catastrophique, la densité du vécu et de l'éprouvé sont telles que les capacités du sujet à y faire face sont débordées. Confronté en direct à la mort (la sienne et/ou celle des autres), avec une soudaineté et d'une intensité extrême, il éprouve des sentiments d'impuissance et d'effroi, dépourvu qu'il est de toute possibilité de représentation, de toute référence préalable et de toute possibilité de maîtrise immédiate de son environnement et de son vécu intérieur.

Pour les proches des victimes, deux cas sont possibles: soit ils sont confrontés à un survivant traumatisé et ils peuvent à leur tour être "contaminés" par leur traumatisme, en particulier les enfants si leur parent reste chroniquement traumatisé, soit il s'agit d'une problématique de deuil si le proche est décédé. 


\section{Tableau 1}

Critères de diagnostic pour le trouble Etat de Stress Post-Traumatique (DSM IV).

A la victime a été exposée à un événement traumatique

B l'événement traumatique est constamment revécu

C évitement persistant de stimuli associés au trauma et émoussement de la réactivité générale

D symptômes persistants d'excitabilité augmentée

E durée supérieure à 1 mois

F le trouble occasionne une détresse cliniquement significative ou une entrave au fonctionnement social, professionnel ou dans toute autre sphère significative de l'existence

\section{Tableau 2}

Epidémiologie du PTSD.

\section{1-3\% de prévalence à vie pour la population en général}

$4 \%$ de prévalence à vie pour les intervenants professionnels (pompiers, policiers, personnel soignant)

jusqu'à 90\% de prévalence à vie pour des victimes gravement maltraitées (prisonniers de guerre et victimes de tortures)

30\%: moyenne de prévalence à vie pour des traumatisés graves (par exemple vétérans du Vietnam)

\section{Principes de prise en charge}

La prise en charge des victimes et la prévention d'une chronicisation de leurs troubles sont donc d'une grande importance. Schématiquement, on peut distinguer trois phases d'intervention dans le respect d'une approche systémique du traumatisé et de sa dimension bio-psycho-sociale.

Au cours de la première phase, la mission des intervenants (en général team psycho-social) doit viser tout d'abord à permettre aux victimes de faire face à la situation immédiate (interne, intrapsychique et externe), de retrouver leur équilibre, de les aider à satisfaire leurs besoins élémentaires et de réintégrer ce qui subsiste de leur cadre existentiel.

La deuxième phase prend place dans les jours qui suivent la catastrophe, et dans tous les cas après une période de récupération physique (repos, réalimentation, reprise du contact avec les proches). Il s'agit alors de permettre la mise en place d'un processus réparateur, qui prendra essentiellement la forme d'un travail de deuil. Nous savons que ce processus évoluera selon les possibilités de chacun, à la vitesse et selon les modalités que leur permet leur organisation psychique.

Le debriefing, maillon important mais aussi très controversé actuellement, a pour but de tenter entre autres d'initier ce processus. Si ses visées préventives, voire même thérapeutiques ont été largement surestimées, il garde tout de même son utilité pour autant qu'il soit conduit par des intervenants bien formés. Il répond également à la demande d'un certain nombre de victimes qui éprouvent le besoin de parler de leur vécu, de comprendre et de trouver un sens à ce qui leur est arrivé, et de trouver la réponse aux nombreuses questions qu'ils se posent.

Etymologiquement, le debriefing est le contraire $\mathrm{du}$ briefing (instructions, directives, séance d'information), c'est à dire littéralement un rapport qui fait suite à une action. C'est donc avant tout un bilan, axé évidemment et avant tout sur les aspects psychologiques de l'événement vécu et/ou de l'intervention effectuée. C'est une technique qui consiste à entreprendre dans un premier temps, si possible en groupe, une reconstitution active de l'événement pour aider, dans un deuxième temps, à identifier les émotions vécues. Ensuite seulement interviennent les liens possibles avec des événements passés, avec l'image de soi et avec les réactions des autres.

Cette intervention a également pour but une abréaction des émotions, de mettre en mots les émotions vécues, d'organiser par la parole la violence éprouvée. Il s'agit de permettre la symbolisation du trauma, du chaos, de la déstructuration vécus par l'approche de la mort.

Car plus on tente d'éviter, de contrôler les souvenirs et les émotions concomitantes, et plus ils reviennent. Le fait de suggérer aux victimes qu'il est permis et même souhaitable d'en parler leur permet de ne plus tenter à tout prix de se l'interdire. Et c'est 
alors seulement qu'elles se permettront et auront la possibilité de pouvoir penser à autre chose. Il s'agit d'un processus assimilable à la catharsis, qui consiste à assumer un destin mauvais pour en faire quelque chose de positif.

Un autre but du debriefing consiste à repérer les sujets à risque pour les conseiller sur l'attitude à adopter en cas de persistance des symptômes et de les aider à surmonter leurs réticences à consulter un psychiatre en cas de nécessité.

\section{Recours au spécialiste}

La consultation spécialisée s'avère nécessaire si des symptômes tels que par exemple troubles du sommeil, anxiété, flash-backs, difficultés de reprise de la vie active, troubles de l'humeur persistent. Ces symptômes ne doivent pas être couverts par des médicaments (notamment somnifères et anxiolytiques) qui entraîneraient rapidement un risque de dépendance, mais être abordés aussi sous un angle psychothérapeutique afin d'aider le sujet à retrouver un sens à son existence après la catastrophe, à surmonter l'horreur et les pertes vécues.

Comme mentionnés précédemment, les intervenants peuvent eux aussi être victimes d'une réaction aiguë à un facteur de stress, mais surtout d'un état de stress post-traumatique par un effet de sommation lié à une exposition très fréquente à des situations catastrophiques et très chargées sur le plan émotionnel. C'est tout d'abord pour eux que le debriefing a été largement utilisé et il peut lui, être effectué par des pairs.

Le diagnostic d'état de stress post-traumatique (PTSD) ne peut être posé qu'après quelques semaines, et la survenue de cette affection n'a que rarement lieu plus de 6 mois après l'événement. Son traitement relève de spécialistes, la meilleure combinaison thérapeutique consistant en une psychothérapie associée à une médication appropriée, souvent à base d'antidépresseurs. La prescription à long terme d'anxiolytiques et de somnifères est contre-productive et contribue surtout à organiser, fixer de nombreux paramètres du trouble.

Il faut savoir que le PTSD peut être un trouble grave souvent associé à des diagnostics de comorbidité:

- drogues et abus d'alcool,

- dépressions majeures,

- trouble panique,

- troubles phobiques,

- anxiété généralisée,

- troubles dysthymiques.

Les tentatives de suicide sont plus nombreuses chez les traumatisés psychiques que dans les groupes de contrôle.

\section{Effets secondaires des catastrophes}

Il convient donc de respecter ces données de base et de faire en sorte que la collectivité touchée puisse gérer ces phases successives et surtout ne pas les escamoter.

Même lorsque la menace immédiate de la catastrophe s'est dissipée, il arrive que persistent l'insécurité, de pair avec l'angoisse, ce qui peut inspirer des dispositions adéquates et nécessaires; mais peut au contraire développer aussi une méfiance croissante envers les pouvoirs publics et les sauveteurs ou conduire à se muer en spectateur anxieux de sa propre santé et de celle d'autrui.

Il n'est pas rare de voir persister, au sein la population sinistrée, une disposition augmentée à l'angoisse pendant des mois, voire même des années si rien n'est fait pour leur venir en aide.

Même si j'ai mentionné 3 catégories de population touchées au début de cet article, je n'aimerais pas terminer sans mentionner que la recherche de boucs émissaires est caractéristique des réactions de la population concernée. Le rôle des autorités, et de tous ceux qui doivent et savent raison garder, consiste à éviter que des innocents ne deviennent à leur tour des victimes indirectes de la catastrophe.

\section{Références}

- Psychiatrie de guerre et de catastrophe: Aide mémoire de l'armée suisse.

- Barrois C. Les névroses traumatiques. Paris: Dunod; 1998.

- Girard R. Le bouc émissaire. Paris: Le Livre de Poche: 1986.

- de Clercq M, Lebigot F. Les traumatismes psychiques. Paris: Masson; 2001

- La Revue Francophone du Stress et du Trauma.

- Journal of Traumatic Stress. 